\title{
Research on the Security Evaluation of Computer System Based on Fuzzy Comprehensive Evaluation Method
}

\author{
Jimin Gao \\ Department of Computer Engineering, Shenzhen Polytechnic, Shenzhen, China \\ gaojm@szpt.edu.cn
}

Keywords: Computer security, Fuzzy comprehensive evaluation, AHP

\begin{abstract}
Information has become an important resource for mankind. In handling these huge amount of information, computer information processing has become indispensable. At present, how to predict and evaluate computer systems security in a better way emerged as a research hot spots. In this paper, fuzzy comprehensive evaluation theory is introduced to solve accident risk evaluation of the computer information system, and its feasibility is demonstrated. A computer network center of a university is taken as an example to describe how to set up a computer information systems security evaluation model, and the evaluation results provide a guide and basis for establish the preventive measures of computer information systems accident.
\end{abstract}

\section{Introduction}

With the wide application and rapid popularization of computer in all fields of society, human society has entered the information age [1,2]. Information has become an important resource for human kind. In a computer system, the information refers to programs and data stored in the computer's internal and external devices. Because information stored in computer system is related to national security in departments such as political, economic and military, and also related to institution, organization and individual confidential[4,5]. It is vulnerable to hostile forces, a number of illegal users and people with ulterior motives of threats and attacks [6]. Therefore, the computer system security issues has been payed more and more attention [7]. At present, how to better predict and evaluate computer systems security situation has become a research hot spot .

\section{Computer Security System Overview}

The computer system also known as computer information systems, is combined by computers and their associated supporting equipment and facilities (including network). According to a certain application objectives and rules, it is a man-machine system for information collection, processing, storage, transmission and retrieval[8]. Computer information system security can be divided broadly into three aspects: physical security, operational security and information security. Since the information is an important strategic resource, computer system is used in centralized information management for enterprise and national politics, military, financial, business, etc. Therefore the computer system has become the main target of criminals. In order to simplify in research and meet the practical needs, this paper focus on the information security.

\section{Fuzzy Comprehensive Evaluation Theory Overview}

Fuzzy comprehensive evaluation is a method for multi-factor events to implement semi-quantitative analysis, which is not suitable for quantitative analysis. Subjective judgment and qualitative description are expressed in form of grades, and system accident risk is determined by fuzzy operation in degree of membership[9]. This method can be used to certain extent to check and reduce the subjective influence of human, so as to make the analysis more scientific. The risk of computer information system is determined by multiple factors, the utilization of fuzzy comprehensive evaluation method can divide the whole system into several units or factors [10]. On the basis of determining the risk degree and weight of each factor, the risk of computer information 
system is comprehensively evaluated.

\section{Computer Information System Accident Risk Security Evaluation}

Security evaluation model of computer information system accident risk. The quantitative description of the computer information systems risk. The computer information systems risk $(R)$ is determined by the occurrence probability of computer information system accident $(P)$ and the danger of accidents $(d)$. Thus, the risk of computer information system accident is the function of accident probability and danger:

$$
R=f(p, d)
$$

Where in due to an accident probability is difficult to calculate for computer information systems, it generally refers to the occurrence of the accident rate of computer information systems, namely the possibility of accidents. Hazards caused by the computer information system accident typically includes information loss, denial of service, communications interference, recovery costs, property damage and social impact, which should include direct and indirect losses.

The comprehensive evaluation model. The model consists of three layers which are composed of two evaluation index, shown in Figure 1.

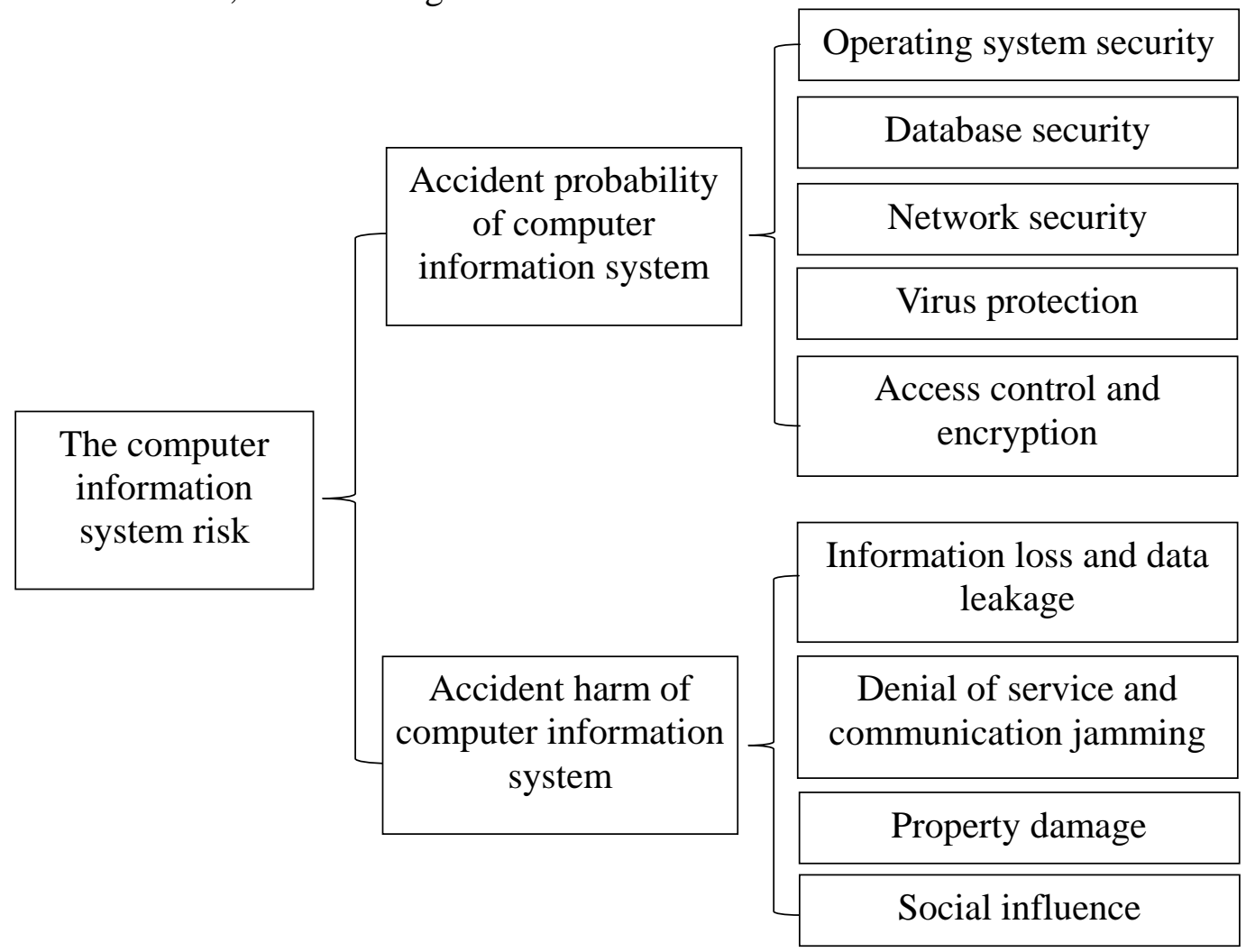

Figure 1. The comprehensive evaluation model

The possibility analysis of computer information systems accidents. A computer network service center of a certain university is taken as an example, and make an evaluation analysis for it, which is shown as follows:

Establish the set of evaluation grades $V=\left\{v_{1}, v_{2}, v_{3}, v_{4}, v_{5}\right\}$. The possibility of computer network center risk accidents can be divided into five levels in turn. $V=\{$ First level, Second level, Third level, Fourth level, Fifth level\}.

According to the specific situation of the proposed computer network center, select four key factors to establish the factors set $U=\left\{u_{1}, u_{2}, u_{3}, u_{4}\right\}$, the specific factors include $U=$ \{Operating system security, Database security, Network security, Virus protection\}.

This paper uses the expert scoring collection method, which consults four categories experts including professors and scholars from universities and research institutes, computer systems administrators, network technicians, and experienced users. Based on their own practical experience 
in using the system, make a score for computer information network system security. Then take a statistical processing for the scoring and obtain the single factor fuzzy relation matrix.

$$
R_{1}=\left[\begin{array}{lllll}
0.12 & 0.16 & 0.18 & 0.18 & 0.36 \\
0.18 & 0.17 & 0.13 & 0.32 & 0.20 \\
0.22 & 0.10 & 0.45 & 0.15 & 0.18 \\
0.35 & 0.20 & 0.15 & 0.17 & 0.13
\end{array}\right]
$$

Determine the weight vector of evaluation factors $A=\left(a_{1}, a_{2}, a_{3}, a_{4}\right)$. Under normal circumstances, the $\mathrm{m}$ evaluation factors are not equally important to evaluation object. The performance of the various factors on the overall performance of the system is also different. Thus, before taking fuzzy transformation, it needs to determine and normalized the evaluation factors weight vector $\boldsymbol{A}$.

Currently, there are many methods to determine the weight of each index. AHP method is an effective method. In this paper, AHP method is used to determine the computer information system accident factor weights. We use the scaling method proposed by Professor Saaty to construct the judgment matrix. And invite experts to evaluate computer network system accident factor, the results are shown in Table 1.

Table 1. The judgment matrix

\begin{tabular}{ccccc}
\hline$A$ & $B_{1}$ & $B_{2}$ & $B_{3}$ & $B_{4}$ \\
\hline$B_{1}$ & 1 & 2 & 3 & 5 \\
$B_{2}$ & $1 / 2$ & 1 & 3 & 4 \\
$B_{3}$ & $1 / 3$ & $1 / 3$ & 1 & 3 \\
$B_{4}$ & $1 / 5$ & $1 / 4$ & $1 / 3$ & 1 \\
\hline
\end{tabular}

Calculate the index weight of factors in principle layer and factor layer which is shown as follows:

First, calculate the production of each line index score in the judgment matrix: $M_{i}=\prod_{j=1}^{4} B_{i j}$, $i=1,2,3,4$;

Second, calculate the fourth root of $M_{i}: \bar{M}_{i}=\sqrt[4]{M_{i}}$;

Third, normalized the vector $\bar{M}_{i}=\left(\bar{M}_{1}, \bar{M}_{2}, \bar{M}_{3}, \bar{M}_{4}\right)$, and obtain the weight vector $A_{1}=\left(a_{1}, a_{2}, a_{3}, a_{4}\right)$.

According to the above steps, the vector $A_{1}=(0.44,0.30,0.14,0.12)$ can be calculated.

Use fuzzy synthetic operator to obtain the evaluation vector. In order to better reflect the role of weights, the operator $M(\vee, \cdot)$ is applied on the main factors.

$$
\begin{aligned}
B_{1} & =A_{1} \cdot R_{1}=\left(\begin{array}{llll}
0.44 & 0.30 & 0.14 & 0.12
\end{array}\right) \cdot\left[\begin{array}{lllll}
0.14 & 0.18 & 0.18 & 0.22 & 0.28 \\
0.18 & 0.17 & 0.13 & 0.42 & 0.10 \\
0.22 & 0.10 & 0.45 & 0.15 & 0.18 \\
0.35 & 0.20 & 0.15 & 0.17 & 0.13
\end{array}\right] \\
& =\left(\begin{array}{lllll}
0.062 & 0.079 & 0.079 & 0.126 & 0.123
\end{array}\right)
\end{aligned}
$$

Normalized the vector $B_{1}$ and obtain $B_{01}=\left(\begin{array}{lllll}0.13 & 0.17 & 0.17 & 0.27 & 0.26\end{array}\right)$, namely the probability membership of first level computer network center accident is 0.13 , the probability membership of second level computer network center accident is 0.17 , the probability membership of third level computer network center accident is 0.17 , the probability membership of fourth level computer network center accident is 0.27 , the probability membership of fifth level computer network center accident is 0.26 . According to the principle of maximum degree of membership, the 
probability of the computer network center accident belongs to the fourth level, namely the possibility of an accident is very high.

The danger analysis of computer information system accident. Ditto, establish the set of evaluation grades $V=\left\{v_{1}, v_{2}, v_{3}, v_{4}, v_{5}\right\}$. The danger of computer network center risk accidents can be divided into five levels in ascending order. $V=\{$ First level, Second level, Third level, Fourth level, Fifth level\}.

According to the specific situation of the proposed computer network center, select three key factors to establish the factors set $U=\left\{u_{1}, u_{2}, u_{3}\right\}$, the specific factors include $U=\{$ Missing data information disclosure, Denial of service communications interference, Loss of property\}. The following can be obtained through expert scoring method.

$$
R_{2}=\left[\begin{array}{lllll}
0.11 & 0.20 & 0.42 & 0.16 & 0.11 \\
0.12 & 0.12 & 0.58 & 0.10 & 0.08 \\
0.14 & 0.25 & 0.41 & 0.11 & 0.09
\end{array}\right]
$$

Use the AHP method to determine evaluation factors weight vector $A_{2}=\left(a_{1}, a_{2}, a_{3}\right)$. It can be obtained:

$$
\begin{aligned}
A_{2} & =\left(\begin{array}{llll}
0.35 & 0.40 & 0.25
\end{array}\right) \\
B_{2} & =A_{2} \cdot R_{2}=\left(\begin{array}{lllllll}
0.35 & 0.40 & 0.25
\end{array}\right) \cdot\left[\begin{array}{ccccc}
0.11 & 0.20 & 0.42 & 0.16 & 0.11 \\
0.12 & 0.12 & 0.58 & 0.10 & 0.08 \\
0.14 & 0.25 & 0.41 & 0.11 & 0.09
\end{array}\right] \\
& =\left(\begin{array}{lllll}
0.048 & 0.070 & 0.232 & 0.056 & 0.038
\end{array}\right)
\end{aligned}
$$

Normalized the vector $B_{2}$ and obtain $B_{02}=\left(\begin{array}{lllll}0.11 & 0.16 & 0.51 & 0.13 & 0.09\end{array}\right)$, namely the danger membership of first level computer network center accident is 0.11 , the danger membership of second level computer network center accident is 0.16 , the danger membership of third level computer network center accident is 0.51 , the danger membership of fourth level computer network center accident is 0.13 , the danger membership of fifth level computer network center accident is 0.13. According to the principle of maximum degree of membership, the danger of the computer network center accident belongs to the third level.

The fuzzy comprehensive evaluation of computer information system accident risk. The accident risk of computer information systems is also divided into five levels, the fuzzy matrix can be calculated by the principle layer calculation results:

$$
R_{3}=\left[\begin{array}{lllll}
0.13 & 0.17 & 0.17 & 0.27 & 0.26 \\
0.11 & 0.16 & 0.51 & 0.13 & 0.09
\end{array}\right]
$$

Experts give weight of possibility and danger of computer information system risk, that is $A_{3}=\left(\begin{array}{ll}0.53 & 0.47\end{array}\right)$, there are

$$
\begin{aligned}
& B_{3}=A_{3} \cdot R_{3}=\left(\begin{array}{ll}
0.53 & 0.47
\end{array}\right) \cdot\left[\begin{array}{lllll}
0.13 & 0.17 & 0.17 & 0.27 & 0.26 \\
0.11 & 0.16 & 0.51 & 0.13 & 0.09
\end{array}\right] \\
& =\left(\begin{array}{lllll}
0.0689 & 0.0901 & 0.2397 & 0.1431 & 0.1378
\end{array}\right)
\end{aligned}
$$

Normalized the vector $B_{3}$ and obtain $B_{03}=\left(\begin{array}{lllll}0.10 & 0.14 & 0.35 & 0.21 & 0.20\end{array}\right)$. Based on the principle of maximum membership, the accident risk of the proposed computer information system belongs to third level.

\section{Conclusion}

In this paper, fuzzy comprehensive evaluation method is used to evaluate the accident risk of computer information system, which is an application of safety engineering for computer security risk assessment. It can make the accident prevention countermeasure of computer information systems more scientific, reasonable and effective. However, the application of fuzzy comprehensive 
evaluation method in computer information system is still in experiment stage, the conclusion can only give us a macroscopic reference, and therefore it needs further development and improvement.

\section{References}

[1] Xue-Min Zhang, Gang-Wei Wang, Conghuan, Ye. Evaluation in food security supervision system based on cloud computing [J]. Advance Journal of Food Science and Technology, 2015, 8(10), 704-706.

[2] Yan Qiang, Shu Hua-Ying, Chen Zhong, Duan Yun-Suo. Object-oriented method for information system security evaluation [J]. Journal of Beijing University of Posts and Telecommunications, 2005, 28(4), 69-73.

[3] Ou Yuyi, Xie Jinbiao, Ling Jie. An improved evaluation index system for the host information security evaluation system [J]. International Journal of Security and its Applications, 2015, 9(3), 49-62.

[4] Guo Jin-Cheng, Fan Dan, Che Hao-Yuan, Duan Yun-Na, Wang Hong-Su, Zhang Da-Wei. An approach to network security evaluation of computer network information system with triangular fuzzy information [J]. Journal of Intelligent and Fuzzy Systems, 2015, 28(5), 2029-2035.

[5] Farn Kwo-Jean, Lin Shu-Kuo, Fung Andrew Ren-Wei. A study on information security management system evaluation - Assets, threat and vulnerability [J]. Computer Standards and Interfaces, 2004, 26(6), 501-513.

[6] Udell C.J., Carlson R.L. Risk Evaluation Syetem For Facility Safeguards And Security Planning [J]. Nuclear materials management, 1987, 16, 50-53.

[7] Guo Jin-Cheng, Fan Dan, Che Hao-Yuan, Duan Yun-Na, Wang Hong-Su, Zhang Da-Wei. An approach to network security evaluation of computer network information system with triangular fuzzy information [J]. Journal of Intelligent and Fuzzy Systems, 2015, 28(5), 2029-2035.

[8] Li Baozhu, Bi Ran. The application of fuzzy-ANP in evaluation index system of computer security [J]. Key Engineering Materials, 2010, 439, 754-759.

[9] Yuan Xiao Tong, Yang Hua Min. The software quality fuzzy comprehensive evaluation method research [J]. Applied Mechanics and Materials, 2014, 513, 1959-1962.

[10] Cao Yi, Zhao Bo, Zhang Lifeng. Research and Realization of Software Quality Fuzzy Co[6] Dong Jianli, Shi Ningguo. Research on fuzzy extension synthesis metrics algorithm for software quality [J]. Journal of Software, 2011, 6(11), 2099-2105. 